\title{
Phase-field simulations of pore migration and morphology change in thermal gradients
}

\author{
Ian W. Vance ${ }^{1}$, Paul C. Millett ${ }^{1 *}$ \\ ${ }^{1}$ Department of Mechanical Engineering, University of Arkansas, Fayetteville, AR 72701 \\ *Correspondence to: pmillett@uark.edu
}

\begin{abstract}
Here we present a phase-field simulation model that captures the thermalgradient-driven migration of pores in oxide fuel associated with fuel restructuring. The model utilizes a Cahn-Hilliard equation supplemented with an advection term to describe the vapor transport of fuel material through the pore interior due to gradients in vapor pressure. Simulations demonstrate that the model not only predicts pore migration towards the centerline of the fuel, but also a concurrent change in pore shape during migration from an initially isotropic morphology to either a lenticular morphology or a prolate morphology depending on the vapor transport conditions. This model is a necessary first step to conducting accurate simulations of the microscopic changes that occur during the complicated process of oxide fuel restructuring.
\end{abstract}




\section{Introduction}

Nuclear reactors operate at high temperatures and the thermal gradients that develop within the fuel can drive unique microstructural changes [1]. In particular, fast reactor oxide fuel exhibits a unique fuel restructuring evolution that occurs early in its lifetime once temperature gradients exceeding $1000 \mathrm{~K} / \mathrm{cm}$ become established. The initial porosity within the as-fabricated fuel $(5-10 \%$ after compaction sintering) undergoes a radially inward migration due to the steep temperature gradients that leads to the formation of a 'central void' [2]. Experimental observations moreover have documented that a migrating pore's shape can transform into either a lenticular morphology or a prolate (bullet-shaped) morphology depending on the radial location of the pore [3], see Fig. 1. This substantial redistribution of porosity alters the grain structure whereby, in the inner one-third of the fuel pellet, the initial equiaxed grain distribution is replaced with larger columnar grains that are inwardly oriented towards the fuel center [2].

The physical origins of thermal gradient pore migration have been considered for decades. Two basic mechanisms have emerged to explain the phenomenon: (i) solidstate thermodiffusion (or Soret-effect diffusion [4]) either at the pore surface or within the solid, and (ii) vapor transport of the solid material across the inside of the pore. In the former mechanism, a temperature gradient acts as a secondary driving force for solidstate diffusion, in addition to the conventional concentration gradient driving force. On the other hand, for vapor transport, the driving force is the variation of the vapor pressure of the solid material with temperature inside the pore. This mechanism requires the evaporation of fuel material on the hot side of the pore and condensation of material on 
the cold side of the pore. The models of Nichols [5,6,7] and of Sens [3] are the most often cited descriptions of the vapor transport mechanism (they are, in fact, quite similar theoretical treatments [8]).

According to theoretical derivations, each mechanism dominates the migration velocity at different regimes of pore size $[1,9]$. For example, the pore velocity due to the thermodiffusion mechanism is inversely proportional to the pore diameter, and therefore conceivably dominates the migration of nanoscale cavities and gas-filled bubbles. Indeed, atomistic simulations have captured thermal gradient pore migration of very small cavities $(\sim 2 \mathrm{~nm})$ and convincingly demonstrated temperature-gradient induced surface diffusion activity [10]. Conversely, according to theory, the pore velocity due to vapor transport is independent of pore diameter, and therefore it is likely the dominant mechanism for the migration of larger pores (> several microns). Simulating the migration of larger pores is not possible with atomistic numerical methods, and it is still exceedingly difficult to experimentally observe such pore migration with in-situ techniques (although experimental studies [11] have reproduced lenticular pore migration in a laboratory setting, with measurements that agree well with Sens' vapor transport model [3]).

Rather, mesoscopic simulation methods can provide important insight for this topic. Both phase-field [12,13,14] and Monte Carlo [15] methods have been developed to simulate pore migration in a temperature gradient. Each of these works incorporated a thermodiffusion model to drive pore migration, despite the fact that theoretical descriptions predict the vapor transport mechanism to be dominant. In these models, thermodiffusion is implemented either with a temperature-dependent free energy function 
$[12,13]$ or with a flux term containing the temperature gradient [14]. Indeed, the simulations produced void migration in the high-temperature direction; however, pore shapes remained unchanged during migration, which is counter to experimental observations that migrating pores transform into highly non-equilibrium geometries (e.g. lenticular or prolate shapes). Computer simulations have yet to capture this drastic change in pore shape during migration. Moreover, a mesoscale simulation model has yet to be developed that describes pore migration due to vapor transport.

Here, we have developed a phase-field model that incorporates the vapor transport mechanism to simulate thermal gradient pore migration in oxide fuel. In contrast to previous mesoscale simulation studies, our model captures both pore migration towards the high temperature region as well as a concurrent change in the pore shape from an isotropic morphology to either a lenticular morphology or a prolate (bullet-shaped) morphology, consistent with experimental observations. The vapor transport mechanism is integrated into the model by adding an advection term to the Cahn-Hilliard equation that describes the transport of $\mathrm{UO}_{2}$ molecules across the interior of the pore. We implement a vapor transport velocity for $\mathrm{UO}_{2}$ as developed by Sens [3] that enters the advection term that drives pore migration. The simulations reveal that the pore shape changes to a lenticular morphology when the transport velocity has a negative slope in the radial direction, which occurs in the innermost regions of the fuel pellet. Alternatively, the pore shape changes to a prolate morphology when the transport velocity has a positive slope in the radial direction, which occurs in the middle regions of the fuel pellet. Hence, we show that the key to pore shape change is the nature of the slope of the vapor transport velocity in the radial direction. Both of these pore shapes, 
and their relative positions in the radial direction, agree with experiments [3]. The predictions made by this phase-field model lend further support to the early models that predict the vapor transport mechanism dominates pore migration evolution during fuel restructuring.

\section{Simulation methodology}

Before describing the phase-field model, we start by discussing the previous theoretical descriptions of vapor-transport pore migration. The derivation of Sens [3] begins with the assumption that vapor pressure within the as-fabricated pores is independent of surface tension and rather only a function of the local temperature (this is primarily justified by the fact that migrating pores are far from spherically shaped, so an equilibrium pressure $2 \gamma / r$ cannot be applied). By solving a diffusion equation describing vapor transport due to gradients in temperature and pressure, and by utilizing an empirical description of $\mathrm{UO}_{2}$ vapor pressure, Sens derived an expression for the transport velocity of gaseous $\mathrm{UO}_{2}$ as a function of temperature and temperature gradient (see Eq. 25 in [3]):

$$
\boldsymbol{v}=A\left(B+C T+D T^{2}+E T^{3}\right) T^{-5 / 2} \Delta H p_{o} \exp \left(-\frac{\Delta H}{R T}\right)\left(\frac{d T}{d r}\right)_{\text {matrix }},
$$

where $T$ is temperature, $\Delta H$ is the enthalpy of vaporization of $\mathrm{UO}_{2}, p_{o}$ is a pressure prefactor, and $A-E$ are empirical constants. Using values for $A-E, \Delta H$, and $p_{o}$ prescribed by Sens, the transport velocity can be plotted versus the fuel radial distance as shown in Fig. 2. Here, the temperature is assumed to conform to a parabolic profile with a centerline value of $2800 \mathrm{~K}$ and an outer value of $900 \mathrm{~K}$ (values that are appropriate for fast reactors rather than LWR reactors). Due to the assumption that vapor pressure is 
independent of surface tension, this velocity profile of $\mathrm{UO}_{2}$ vapor also represents the pore velocity as a function of radial position. Note that the velocity is negative corresponding to a migration direction from right to left on the $\mathrm{x}$-axis (i.e., towards the fuel centerline given that the positive $\mathrm{x}$-direction points from the fuel center to fuel periphery).

Conventional phase-field models describe mass transport and interface migration by solid-state diffusion processes as specified by the Cahn-Hilliard equation [16]:

$$
\frac{\partial c}{\partial t}=\nabla \cdot(M \nabla \mu),
$$

where $c$ is a conserved variable often representing the concentration of a chemical species, $M$ is the mobility of that species, and $\mu$ is the chemical potential of that species defined by a Ginzburg-Landau energy functional:

$$
\mu=\frac{\delta F}{\delta c}=\int\left(f^{\prime}(c)-\kappa \nabla^{2} c\right) d V,
$$

where $f^{\prime}(c)$ is the derivative of a free energy density associated with that chemical species.

On the other hand, mass transport of a dissolved species within a convecting gas or liquid is known as advection and is represented as

$$
\frac{\partial c}{\partial t}=-\nabla \cdot(\boldsymbol{v} c),
$$

where $c$ is again a conserved variable and $\boldsymbol{v}$ is the velocity field of a fluid that is transporting the dissolved species.

Therefore, to simulate the vapor transport mechanism governing pore migration, we propose the following Cahn-Hilliard diffusion-advection equation:

$$
\frac{\partial c}{\partial t}=\nabla \cdot(M \nabla \mu)-\nabla \cdot(\boldsymbol{v} c) .
$$


Here, the velocity field $\boldsymbol{v}$ can be assigned the expression derived by Sens for the vapor transport velocity, as shown in Eq. (1). We assume the variable $c$ represents a vacancy concentration in the $\mathrm{UO}_{2}$ material and also that a pore is a cluster of vacancies such that within a pore $c \approx 1$ [17]. We take advantage of the inherent ability of the Cahn-Hilliard model to evolve interfacial migration within multi-phase microstructures by utilizing a simple double-well free energy density expression $f(c)=c^{2}(1-c)^{2}$ with local minima at $c=0$ and $c=1$. We acknowledge that more physics-based expressions may be used for $f(c)$, for example a regular solution model $[17,18]$. The emphasis of this paper however is to explore the combined mechanisms of diffusion and advection with a directional driving force that leads to the one-dimensional migration of a secondary phase, in this case a void.

Two important points regarding the above model require further discussion. First, our expression for the free energy density includes two minima at $\mathrm{c}=0$ and $\mathrm{c}=1$, the latter corresponding to the pore phase. The condition that $f^{\prime}(c=1)=0$ does not restrict the pore phase to have a vacancy concentration of exactly one. Certain driving forces can drive the concentration away from this equilibrium value, and in the current case, the advective flux achieves this result so that the pore is not composed completely of vacancies, but rather contains some solid matter undergoing advection within the pore. Second, our model does not explicitly treat the rate of atomic evaporation or condensation at the pore surfaces. Of course, these processes must occur for pore migration to take place (and they do occur implicitly in our simulations as concentration is transported to or away from the surfaces); however, they are not assumed to be ratelimiting with respect to the pore migration. This assumption was also made by Sens, 
whose model postulates that the rate-limiting mechanism is vapor transport and that evaporation/condensation processes occur as needed to maintain local vapor pressure equilibrium near surfaces [3].

We solved Eq. (5) using the semi-implicit spectral method [19] for the diffusion term (the first term on the right-hand side) and a central finite difference approximation for the advection term. Two-dimensional simulations were executed in which a uniform rectilinear grid with $512 \times 128$ nodes (or in some cases $1024 \times 128$ nodes) was used for discretization. In order to minimize the impact of round-off errors, reduced units for length and time were chosen to execute the simulations, in which the grid spacing parameters $\Delta \mathrm{x}=\Delta \mathrm{y}=1.0$. To revert to physical lengths, these units correspond to $\Delta \mathrm{x}=$ $\Delta \mathrm{y}=500 \mathrm{~nm}$, therefore the simulation boxes represent $256 \times 64 \mu \mathrm{m}$ (or for the larger domains $512 \times 64 \mu \mathrm{m}$ ) regions within the fuel. The $\mathrm{x}$-direction corresponds to the radial direction, and this is the direction along which the velocity field gradient is present. We used a time step value of $\Delta t=0.1$ reduced units; the reduced time unit corresponding to 40 seconds.

In the simulations, the imposed transport velocity field is also represented in reduced units. Two different velocity field profiles were implemented for two different sets of simulations:

$$
\begin{aligned}
& v_{x}=-0.012 x \\
& v_{x}=0.005 x-3.1
\end{aligned}
$$

and $v_{y}=0$ throughout the domains. These $\mathrm{x}$-direction velocity fields are linear functions that have negative values throughout the domain (hence pores always travel in the negative radial direction), but one has a negative slope and the other has a positive slope. 
These two velocity profiles represent the two different shaded regions in Fig. 2. When these two linear velocity profiles are re-scaled into physical units of centimeters and seconds, they can be plotted and compared with Eq. (1) - Sens' model - as is shown in Fig. 3. Equation (6) overlaps well in the innermost region of the fuel radius, hence simulations with this velocity profile correspond with radial distances from 0.0 to 0.0256 $\mathrm{cm}$ in the radial direction. On the other hand, simulations using Eq. (7) correspond with the window from 0.185 to $0.2106 \mathrm{~cm}$ in the radial direction (note that in Fig. 3, we shifted Eq. (7) to the right by a distance of $0.2 \mathrm{~cm}$ while keeping the slope constant). All simulations are run for 3000 time steps.

\section{Results and Discussion}

The simulations presented here characterize the migration of a single pore with an initially circular shape. Figure 4 depicts successive snapshots of a pore as it migrates across the domain in the $\mathrm{x}$-direction. This particular simulation was run with a $1024 \times 128$ grid (i.e., the larger grid) with the velocity profile specified by Eq. (6). The pore has an initial diameter of $30 \Delta \mathrm{x}$, or in physical units $15 \mu \mathrm{m}$, and its center is initially located at $\mathrm{x}$ $=900 \Delta x$. When using the velocity profile of Eq. (6), the simulation domain corresponds to a region adjacent to the centerline of the fuel, in which the left side of the domain is the fuel centerline and the right side of the domain is $512 \mu \mathrm{m}$ into the radial direction. Due to the fact that the $\mathrm{x}$-component of the velocity field is negative throughout the domain, the pore migrates in the negative $\mathrm{x}$-direction (in a similar spirit to the axial definitions of Fig.

2). More notably, the pore shape transforms from a circle to a lenticular shape during the migration process. This morphology change is a direct result of the slope of the transport 
velocity in the $\mathrm{x}$-direction. The velocity profile is negative and also has a negative slope throughout the domain, therefore the leading surface of the pore experiences a lower (or, less negative) velocity than the trailing surface. As a result, the width of the pore in the $\mathrm{x}$-direction reduces due to the fact that the trailing surface migrates faster than the leading surface. All the while, the concentration parameter $c$ remains strictly conserved, and the volume of the pore is also conserved. This kinetic driving force for pore shape change is countered by the increase in surface area of the pore, hence the pore's width in the $\mathrm{x}$ direction does not reduce to zero. Rather, the pore assumes a steady-state lenticular morphology.

Each of the snapshots in Fig. 4 represent equally spaced intervals of time, with the top image corresponding to the beginning of the simulation. By inspection of the relative position of the pore at each instance of time, it can be deduced that the pore velocity is highest at the beginning of the simulation, while it is on the right side of the domain. In the later stages of the simulation, as the pore migrates to the left side of the domain, its velocity decreases. This is again due to the profile of the velocity field defined by Eq. (6), which is a negative-sloped line that equals zero at the left side of the domain. Hence, the overall pore velocity decreases as the pore approaches the left side of the domain.

To better demonstrate the spatiotemporal evolution of both the pore shape and the advection flux, in Fig. 5 we plot a close-up view of the pore using a contour field representing the vacancy concentration as well as an overlaid vector field representing the advection flux. Figures $5 a-5 c$ show the pore at increasing instances of time during its translation, and Fig. 5d shows a close-up view of the shaded box in Fig. $5 \mathrm{~b}$ to better visualize the advection flux field. The advection flux only exists to any significance 
within the pore interior as can be seen by the length of the vectors. These vectors represent the flux of vacancies within the pore, which are equal but opposite to the flux of $\mathrm{UO}_{2}$ material that occurs from the hot surface to the cold surface.

We were interested to see if the change in the pore shape resulted in a deviation of the pore velocity from the prescribed transport velocity equation. To address this question, we calculated the instantaneous position of the pore's 'center of mass' by averaging the $\mathrm{x}$ - and $\mathrm{y}$-positions of all the grid points with a value of $c>0.5$. These positions were plotted versus simulation time, which subsequently allowed us to calculate the pore's instantaneous velocity using a finite-difference approximation for the derivative of the pore position versus time curve. We then plotted the pore velocity versus the pore's x-position (i.e., the pore's radial position), with the results shown in Fig. 6. Aside from an initial acceleration that the pore must perform (the pore initially has a velocity of zero), the pore's velocity very closely matches the imposed transport velocity model, regardless of the fact that the pore has drastically changed its shape during migration. This result is in agreement with the work of Nichols [7], who concluded that the velocity of a migrating pore due to the vapor transport mechanism does not depend on the pore's shape.

Next, we prescribed the transport velocity expression given in Eq. (7), and Fig. 7 shows snapshots depicting the pore migration and morphology evolution. As opposed to the previous conditions, here the transport velocity consists of negative values throughout the domain (hence, as before, the pore migrates in the negative $\mathrm{x}$-direction), however the velocity field has a positive slope in the $\mathrm{x}$-direction. This velocity field therefore induced a morphology change from a circular shape to a prolate (bullet) shape, which can be 
justified by the fact that the leading pore surface experiences a larger (i.e., a more negative) velocity than the trailing pore surface. This bullet-shaped pore continues to evolve in shape as it migrates further towards the left-hand side of the domain. We observe that a somewhat circular 'head' develops at the leading surface of the pore, which seems to persist during migration. As before, we calculated the pore's velocity throughout the simulation, and plotted it versus the radial direction (Fig. 8), and again we observe that the change in pore shape does not result in a deviation in velocity from the prescribed model.

The dimensional changes observed in the migrating pores were calculated for both transport velocity profiles in terms of the pore's width in both the x-direction and ydirection. Figure 9 shows these values plotted versus the pore's radial position. For both cases, the pores begin in the same position and with a radius of $15 \mu \mathrm{m}$. At the end of the simulation using Eq. (6), the lenticular pore reached x- and y-dimensions of 10.5 and 31.5 $\mu \mathrm{m}$, respectively. At the end of the simulation using Eq. (7), the bullet-shaped pore reached $\mathrm{x}$ - and $\mathrm{y}$-dimensions of 30.0 and $9.5 \mu \mathrm{m}$, respectively. Once again, the only difference between the two simulations is the prescribed values for the transport velocity, and hence this important parameter (and its radial profile, including the sign of its slope) seems to dictate the change in pore shape during migration.

\section{Conclusions}

In summary, a phase-field model is presented to simulate pore migration due to the vapor transport mechanism of $\mathrm{UO}_{2}$ molecules through the pore interior due to a temperature gradient. The model utilizes an advection term that is appended to a 
conventional Cahn-Hilliard equation, within which a vapor transport velocity field must be supplied. Here, we used two simple linear velocity profiles that closely correlate to the transport velocity equation developed by Sens [3] at two different radial positions within the fuel. Our simulations illustrate that the highly non-equilibrium shapes observed in migrating pores, namely lenticular and prolate geometries, can be explained by the slope of the transport velocity field in the radial direction. This gives additional support to the notion that large pore migration during fuel restructuring is driven by the vapor transport mechanism. In addition, we found that the magnitude of a migrating pore's velocity due to the vapor transport mechanism is independent of any changes that occur in the pore's shape. This conclusion agrees with that of Nichols [7], however, may be affected by the assumption built into Sens' model that the internal vapor pressure is solely a function of temperature - thus neglecting contributions from the surface tension. The fact that the pore migration in the current simulations is entirely driven by the Sens model of vapor transport implies that this simulation model is subject to the same assumptions and potential inaccuracies of the Sens model.

Nevertheless, the successful reproduction of the transformation of an initially isotropic pore into a lenticular shape or a prolate shape is an advance over recent attempts to simulate thermal-gradient-driven pore migration $[13,14,15]$. Further refinement of this simulation model could include a direct calculation of the spatially-dependent temperature field surrounding the pore, and allow that to inform the transport velocity equation (here, we assumed a particular temperature profile when we assigned the transport velocity field). Such an effort would then allow one to directly calculate local values of vapor transport velocity to place in Eq. (5) based on local values of temperature 
and temperature gradient rather than directly using the Sens model, thus allowing a validation (or invalidation) of the Sens model. In addition, the migration of multiple pores could be simulated in a polycrystalline matrix to understand the formation of the columnar grain structure. We leave these efforts to future studies.

\section{Acknowledgments}

We acknowledge financial support from the Idaho National Laboratory, and fruitful discussions with Yongfeng Zhang and Michael Tonks. This research was also supported by the Arkansas High Performance Computing Center which is funded through

multiple National Science Foundation grants and the Arkansas Economic Development Commission. 


\section{References}

[1] Olander DR. Fundamental Aspects of Nuclear Reactor Fuel Elements. Oak Ridge, TN: Technical Information Center, Energy Research and Development Administration, 1976.

[2] MacEwan JR, and Lawson VB. "Grain Growth in Sintered Uranium Dioxide: II, Columnar Grain Growth." Journal of the American Ceramic Society 45 (1962) 4246.

[3] Sens PF. "The Kinetics of Pore Movement in UO2 Fuel Rods." Journal of Nuclear Materials 43 (1972) 293-307.

[4] Platten JK. "The Soret Effect: A Review of Recent Experimental Results." Journal of Applied Mechanics 73 (2006) 5.

[5] Nichols FA. "Theory of columnar grain growth and central void formation in oxide fuel rods." Journal of Nuclear Materials 22 (1967) 214-222.

[6] Nichols FA. "Pore migration in ceramic fuel elements" Journal of Nuclear Materials 27 (1968) 137.

[7] Nichols FA. "On the diffusional mobilities of particles, pores and loops" Acta Metallurgica 20 (1972) 207.

[8] Nichols FA. "On the thermal gradient migration of lenticular voids" Journal of Nuclear Materials 84 (1979) 319.

[9] Speight MV. "The migration of gas bubbles in material subject to a temperature gradient" Journal of Nuclear Materials 13 (1964) 207.

[10] Desai TG, Millett PC, Tonks M, and Wolf D. "Atomistic Simulations of Void Migration under Thermal Gradient in UO2." Acta Materialia 58 (2010) 330-39.

[11] Kawamata H, Kaneko H, Furuya H, Koizumi M. "Migration Rate of Lenticular Voids in UO2 Under the Influence of Temperature Gradient." Journal of Nuclear Materials 68 (1977) 48-53.

[12] $\mathrm{Hu} \mathrm{SY}$, Henager $\mathrm{CH}$. "Phase-field simulation of void migration in a temperature gradient.” Acta Materialia 58 (2010) 3230-3237.

[13] Li Y, Hu SY, Sun X, Gao F, Henager CH, Khaleel M. "Phase-field modeling of void migration and growth kinetics in materials under irradiation and temperature field." Journal of Nuclear Materials 407 (2010) 119-125. 
[14] Zhang L, Tonks MR, Millett PC, Zhang Y, Chockalingam K, Biner SB. "Phasefield Modeling of Temperature Gradient Driven Pore Migration Coupling with Thermal Conduction." Computational Materials Science 56 (2012) 161-65.

[15] Tikare V, Holm EA. "Simulation of Grain Growth and Pore Migration in a Thermal Gradient." Journal of the American Ceramic Society 81 (2005) 480-84.

[16] Chen LQ. "Phase-Field Models for Microstructure Evolution." Annual Review of Materials Research 32 (2002) 113-40.

[17] Rokkam S, El-Azab A, Millett PC, Wolf D. "Phase field modeling of void nucleation and growth in irradiated metals" Modelling and Simulation in Materials Science and Engineering 17 (2009) 064002.

[18] Millett PC, El-Azab A, Rokkam S, Tonks M, Wolf D. "Phase-field simulation of irradiated metals, Part I: Void kinetics” Computational Materials Science 50 (2011) 949-959.

[19] Chen LQ, Shen J. "Applications of Semi-implicit Fourier-spectral Method to Phase Field Equations." Computer Physics Communications 108 (1998) 147-58. 


\section{FIGURES}

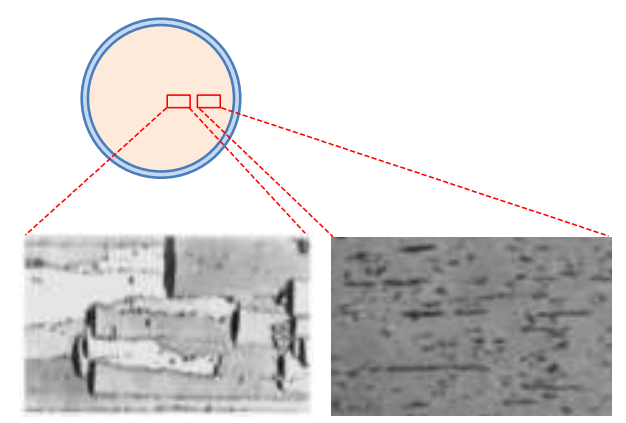

Fig. 1 Microscopy images of both lenticular pores and prolate (bullet) shaped pores during thermal-gradient-driven migration in nuclear fuel. Generally, observations show that lenticular pores develop in the inner regions of the pellet and prolate pores develop at the circumference of the columnar grain region, in the middle or outer sections of the pellet. 


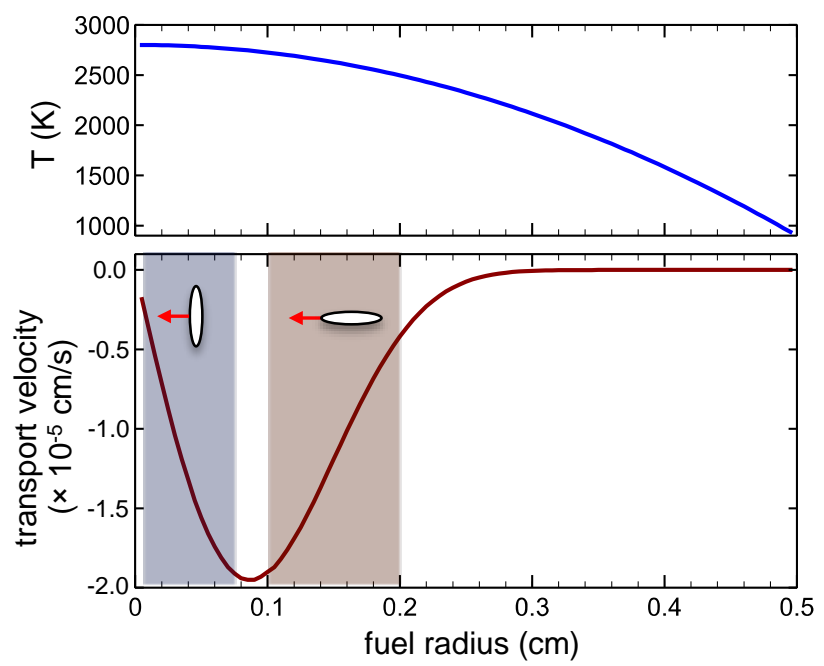

Fig. 2 With an assumed temperature profile in the radial direction (top), the transport velocity of a pore due to the evaporation-condensation mechanism in the radial direction, as derived by Sens [3], is plotted (bottom). Note that in these plots, the positive radial direction is along the $\mathrm{x}$-axis, and a negative transport velocity results in a pore migration toward the fuel centerline. 

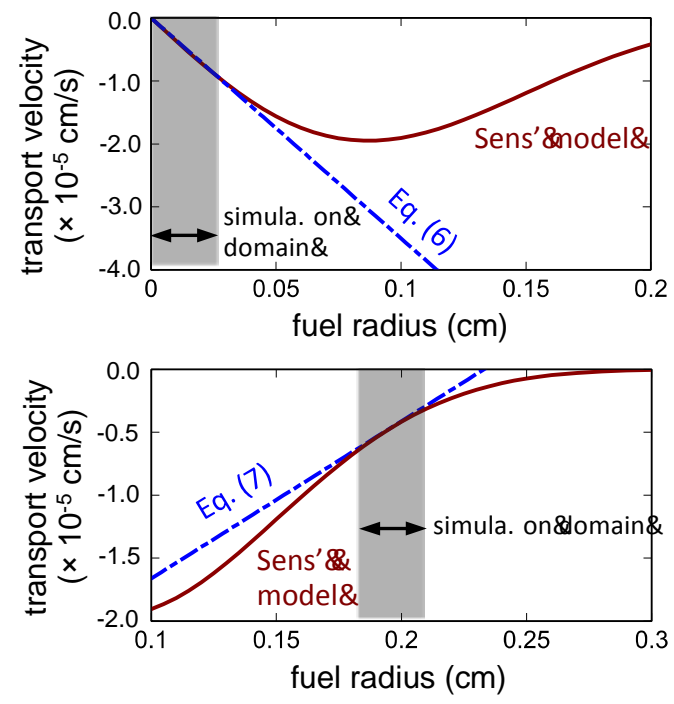

Fig. 3 The simulations in this work used linear equations to describe the transport velocity in the radial direction. These linear equations overlap with the Sens' model [3] at different radial locations, with the top plot showing Eq. (6) and the bottom plot showing Eq. (7). The grey shaded boxes correspond with the simulation domain size in the radial direction $(=256 \mu \mathrm{m}$, with a $512 \times 128$ grid $)$. 
Fig. 4 Simulation snapshots of pore migration using Eq. (6) as the transport velocity field. The top-to-bottom images correspond to 0, 1000, 2000, and 3000 simulation steps, respectively. The left side of the domain corresponds to the fuel centerline when using Eq. (6). These images were taken from a simulation that used the larger grid $(1024 \times 128)$. 


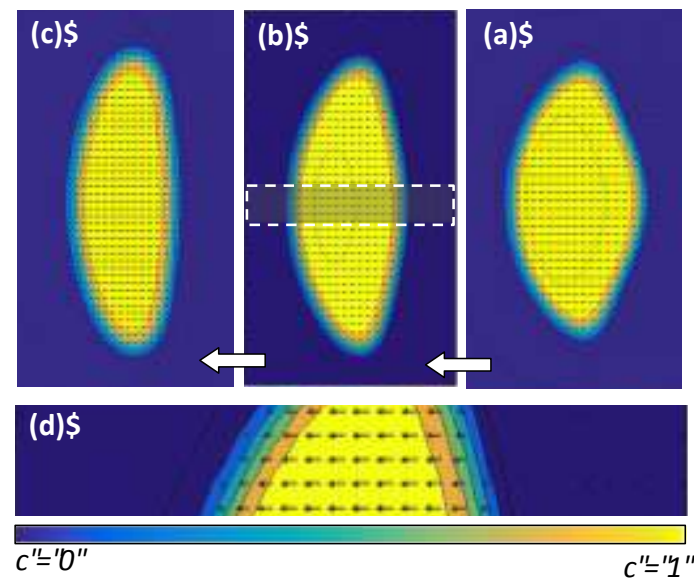

Fig. 5 Contour plots illustrating the pore shape at different instances of time as well as an overlaid vector plot that depicts the advection flux field due to vapor transport. The magnified view in (d) corresponds to the shaded box in (b). Note that the length of the vectors are proportional to the magnitude of flux, and that the flux is only significant within the pore. 


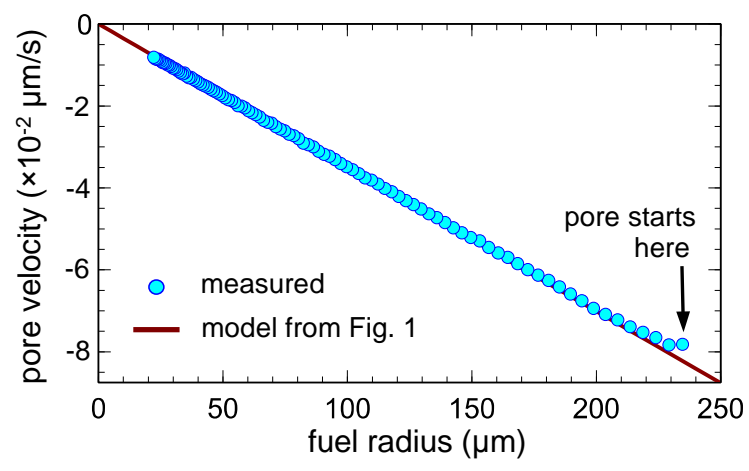

Fig. 6 The velocity of a migrating pore is plotted versus the pore's radial position. The blue data points correspond to velocity values measured from a simulation, as explained in the text, using Eq. (6) for the transport velocity and a grid size of $512 \times 128$. Regardless of the fact that the pore has changed shape to a lenticular morphology, the pore velocity matches very closely to the prescribed model. 
Fig. 7 Simulation snapshots of pore migration using Eq. (7) as the transport velocity field. The top-to-bottom images correspond to 0, 1000, 2000, and 3000 simulation steps, respectively. These images were taken from a simulation that used the larger grid $(1024 \times 128)$. 


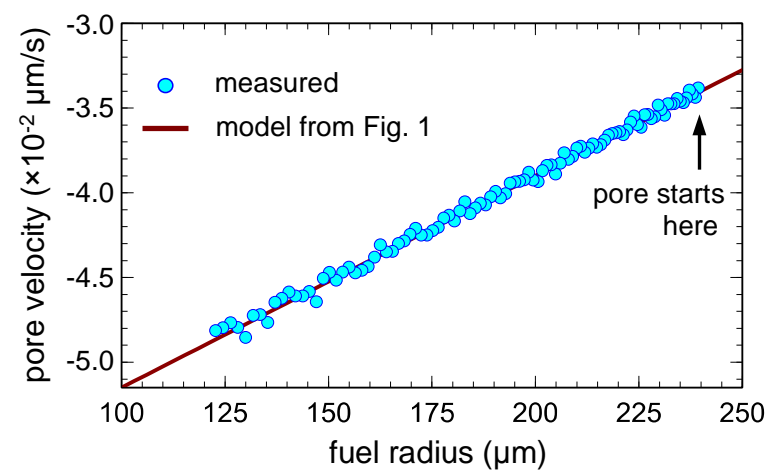

Fig. 8 The velocity of a migrating pore versus the pore's radial position for a pore that transforms into a prolate (e.g., bullet) shaped morphology as shown in Fig. 6 when Eq. (7) is used as the transport velocity. 

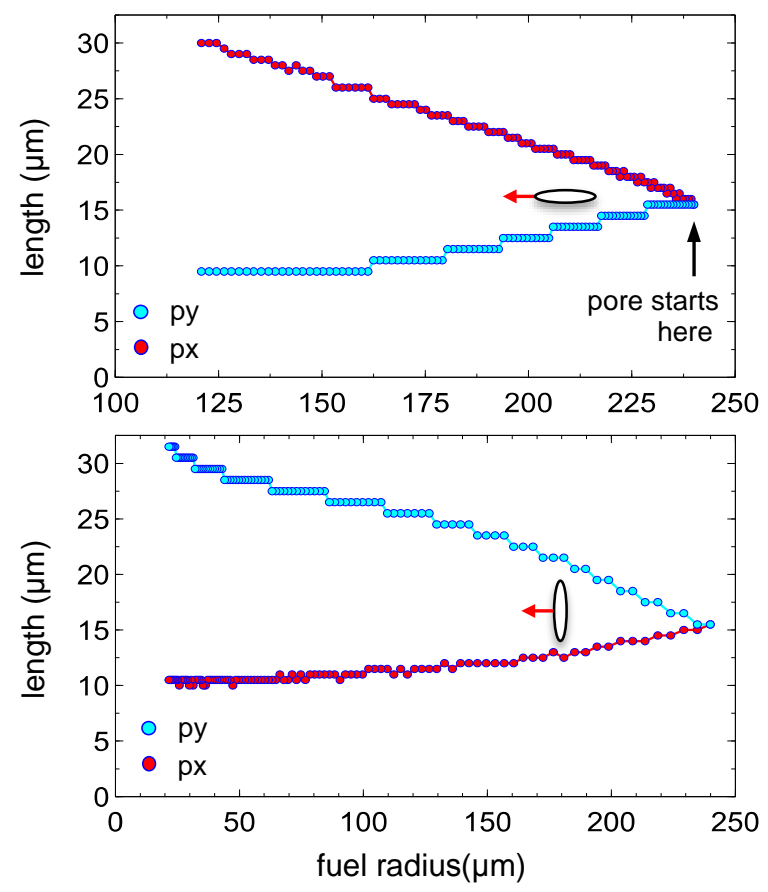

Fig. 9 The $x$ - and y-dimensions of a migrating pore that transforms into (top) a prolate morphology and (bottom) a lenticular morphology. These values were measured from simulations using Eq. (7) and Eq. (6) for the top and bottom plots, respectively. The blue dots correspond to the pore's y-dimension and the red dots correspond to the pore's xdimension. 